\title{
FVIII, Protein C and the Risk of Arterial Thrombosis: More than the Sum of Its Parts
}

\author{
Bob Siegerink ${ }^{1,2}$ \\ ${ }^{1}$ Center for Stroke Research Berlin, Charité Universitätsmedizin \\ Berlin, Berlin Germany \\ 2 Department of Clinical Epidemiology, Leiden University Medical \\ Center, Leiden University, Leiden, the Netherlands
}

Thromb Haemost 2018;118:1127-1129.
Address for correspondence Bob Siegerink, PhD, Center for Stroke Research Berlin, Charité Universitätsmedizin, Berlin, Germany (e-mail: bob.siegerink@charite.de).
In this edition of Thrombosis and Hemostasis, Zakai et al present data from the REGARDS study assessing the interplay between two well-established thrombotic risk factors in relation to both stroke and coronary heart disease (CHD) risk. ${ }^{1}$ Their data show that high levels of coagulation factor VIII (FVIII) increase the risk of both stroke and CHD, seemingly more pronounced for the latter. Low levels of protein $\mathrm{C}$ (PC) only seem to increase the risk of CHD as the risk of stroke does not seem to be affected. This in itself is already an interesting find, as it is in contrast with a recent review and analyses of the literature that suggested that an increased coagulation propensity was strongly associated with the risk of stroke than with myocardial infarction, especially in the young. ${ }^{2}$ But the paper by Zakai et al takes the analyses further and puts a focus on the synergistic inter-play between FVIII and PC.

FVIII and PC, pars pro toto, can be seen as markers of the haemostatic balance which is the result of all thrombotic and anti-thrombotic traits. Given the direct effect of activated PC on FVIII, it is not inconceivable that a combination of these two risk factors play a synergistic role in developing arterial thrombosis. ${ }^{3}$ Basically, they ask themselves, is the combination of their effects more than the sum of their parts? Such an inter-play of two risk factors is also known as interaction of which two types can be discerned, being statistical and biological interaction. Each concept of interaction comes with its own applications and interpretations.

Testing for statistical interaction is classically done by including a cross-product term in a regression model and testing for statistical significance. The main goal of such an addition is to optimize the way the model is fitted to the underlying data and is therefore often a good idea when optimizing model fit is the goal of the analyses. When the cross-product term is statistically significantly different from the null hypothesis, there is said to be statistical interaction. However, it is important to note that this inter-

received

June 4, 2018

accepted

June 4, 2018

action is modelled on the scale of the regression model in which the cross-product term is entered. For linear regression, this means that the cross-product term shows the increase of the outcome parameter if one is exposed to both risk factors on top of the sum of the individual effects. However, when relative risks are modelled (e.g. hazard ratios from a Cox proportional hazards model), the scale is multiplicative and therefore the cross-product term shows the increase of the risk on top of the multiplication of the two individual effects. This is why statistical significance of a positive cross-product term is formally referred to as supramultiplicative interaction.

This is distinct from assessing biological interaction, which looks for interaction on an additive scale. The easiest way to assess biological interaction between two risk factors (A and $B$ ) is to estimate and compare the risk of disease for those who are exposed to no risk factor $(-\mid-)$, risk factor $\mathrm{A}(+\mid-)$, risk factor $\mathrm{B}(-/+)$ or the combination of both $(+/+)$. This way, it is possible to model the additional risk conferred by being exposed to both risk factors on top of the risk of the two individual risk factors in itself, also known as supra-additive interaction.

The presence of supra-additive interaction helps to understand the biology of the disease. It indicates that some individuals only develop the disease because they are exposed to the combination of both risk factors. Sometimes it could also hint towards a clinical opportunity to reduce the risk in specific sub-group of patients. This is, however, not always the case as can be learned from the interaction between oral contraceptive (OC) use and high levels of kallikrein C1 (KAL-C1) inhibitor antigen levels. ${ }^{4} \mathrm{OC}$ use alone increases the risk of ischaemic stroke in young women by approximately twofold, whereas KAL- $\mathrm{C} 1$ alone increases the stroke risk by fivefold. The combination of both increases the ischaemic stroke risk by approximately 23 -fold, which is much more than can be expected based on the increase in risk of the two individual risk factors alone. Given that OC use

(c) 2018 Georg Thieme Verlag KG Stuttgart · New York
DOI https://doi.org/ 10.1055/s-0038-1661373. ISSN 0340-6245. 
is a modifiable risk factor, one might wonder whether screening for KAL-C1 before OC use might actually be a good idea. This is, however, not the case, as approximately 15,000 women need to be screened for KAL-C1 of which subsequently $10 \%$ or 1,500 should refrain from starting OC use to prevent one stroke per year. The reason for the need to screen this high number of women is the very low absolute risk of ischaemic stroke in young women. So even though biological interaction analyses might point towards a very strong supra-additive interaction, the clinical usefulness of this knowledge of such a strong synergy is not always specified.

It is key to understand the differences between statistical and biological interaction, as their application and goals can vary quite a bit. Also, the two concepts are not fully complementary or antagonistic. A certain biological interaction might not be picked up when one goes looking for statistical interaction, as supra-additive interaction might actually be just as strong as exact multiplicative interaction. If so, the cross-product term will not be statistically significant, and a relevant biological interaction will go undetected. Also, statistical interaction analyses are often focused on hypothesis testing by comparing the $p$-value of the product term to a pre-set $\alpha$. In general, hypothesis testing alone is seen as an inadequate approach, especially when it is possible to provide a point estimate and corresponding confidence intervals. ${ }^{5}$ There are different methods to quantify the extent of the biological interaction, each with its own interpretation and idiosyncrasies (please refer to de Mutsert et $\mathrm{al}^{6}$ for a general overview and Li and Chambless ${ }^{7}$ for their application within the framework of cox regression modelling). But there are also other issues to consider. For example, assessment of biological interaction required the use of binary, or at least categorical, data. This need, as well as the need to combine these categories in the interaction analyses, is likely to lead to a loss of information as well as a reduction of statistical power. As a consequence, broader categories might be chosen which then might impact the interpretation of the results even further. But sometimes the differences between the two methods are not so relevant as the conclusions that can be drawn from both methods can converge under certain circumstances. For example, when both risk factors increase the risk of outcome, and the product term analyses suggest supra-multiplicative interaction effect, it follows that there is also a supra-additive interaction present.

Let us return to the paper of Zakai et al. Their initial focus seems to lie on statistical interaction as their analyses are centred around the cross-product term. However, their interpretation seems to be more focused on the biology. When this was pointed out during peer review, the authors promptly sent the results of additional analyses assessing the biological interaction. These numbers indicate a strong supra-additive interaction: compared with no risk factor, individuals with high FVIII alone ( $>$ 80th percentile) had a 1.7-fold increase in CHD risk, whereas low PC was only associated with a 1.3 -fold increase in CHD risk. Being exposed to both risk factors, however, was associated with a sevenfold increase in risk. This increase in risk is much more than can be expected based on additive interaction. Further quantification of this increase shows that the relative excess in risk due to interaction was 5.0, suggesting that approximately $72 \%$ of the risk observed in patients with high FVIII in fact can be attributed to the combined effect of high FVIII and low PC (attributable proportion). These numbers also show that the combined effect is approximately six times larger than the individual effects (synergy index).

Why then are the analyses for biological interaction not presented in the final version of the manuscript? The authors had some strong arguments to do so. First of all, the primary analyses was to estimate the increase in CHD risk per standard deviation change (increase for FVIII, decrease for PC), and interaction of continuous exposures can only be assessed on a multiplicative scale. Assessing biological interaction would mean changing the main analyses, with a potential loss of power. Second, the analyses by Zakai et al were based on a case-cohort setting with extensive adjustment for confounding. Unfortunately, not all methodologies to quantify additive interaction have been tried and tested for that particular study design. But most importantly, their analyses indicate an interaction on the multiplicative scale and therefore also provide evidence of interaction on the additive scale. All in all, the authors have understandably decided that their main message, that is, that there is a strong interaction between a very low PC level and higher FVIII levels with regard to CHD risk, was best conveyed by showing the results from a product term analyses.

Where do we go from here? The discussed paper, as well as many other publications, suggests that the ultimate effect of the haemostatic balance is not just the simple sum of its parts. To understand and perhaps predict thrombotic risk in more detail and precision, the interaction between risk factors needs to be taken into account with the appropriate methodology. But even though the different methods to do so are clear and simple in theory, the reality can be more complicated and stubborn as is demonstrated by the paper from Zakai et al. On top of that, assessing the interaction between two risk factors in this era of precision medicine is only the tip of the iceberg. Imagine the complexity of the methodological considerations when assessing the interaction between not 2 but 20 risk factors. So, even though advancements in technology and higher precision in phenotyping might be seen as hallmarks of this era of precision medicine, the proper application and interpretation of existing as well as the development of new methodologies is perhaps more relevant.

Especially, if we want to understand how and why a sum can be more than its parts.

\section{Conflict of Interest}

None.

\section{References}

1 Zakai NA, Judd SE, Kissela B, Howard G, Safford MM, Cushman M. Factor VIII, Protein C and Cardiovascular Disease Risk: The REasons for Geographic and Racial Differences in Stroke Study (REGARDS). Thromb Haemost 2018;118(07):1305-1315 
2 Maino A, Rosendaal FR, Algra A, Peyvandi F, Siegerink B. Hypercoagulability is a stronger risk factor for ischaemic stroke than for myocardial infarction: A systematic review. PLoS One 2015;10: e0133523

3 Ten Cate H, Hackeng TM, García de Frutos P. Coagulation factor and protease pathways in thrombosis and cardiovascular disease. Thromb Haemost 2017;117(07):1265-1271

4 Siegerink B, Govers-Riemslag JWP, Rosendaal FR, Ten Cate H, Algra A. Intrinsic coagulation activation and the risk of arterial thrombosis in young women: results from the Risk of Arterial Thrombosis in relation to Oral contraceptives
(RATIO) case-control study. Circulation 2010;122(18): 1854-1861

5 Vandenbroucke JP, von Elm E, Altman DG, et al; STROBE initiative. Strengthening the Reporting of Observational Studies in Epidemiology (STROBE): explanation and elaboration. Ann Intern Med 2007;147(08):W163-W194

6 de Mutsert R, Jager KJ, Zoccali C, Dekker FW. The effect of joint exposures: examining the presence of interaction. Kidney Int 2009;75(07):677-681

7 Li R, Chambless L. Test for additive interaction in proportional hazards models. Ann Epidemiol 2007;17(03):227-236 